\title{
Intestinal fatty acid-binding protein, a biomarker of intestinal barrier dysfunction, increases with the progression of type 2 diabetes
}

\author{
Yifeng Wang ${ }^{1,2}$, Licheng Ding ${ }^{3}$, Jiayue Yang ${ }^{4}$, Lijun Liu ${ }^{\text {Corresp., } 1}$, Liang Dong ${ }^{\text {Corresp. } 5}$ \\ ${ }^{1}$ Department of Emergency and Critical Care Medicine, Second Affiliated Hospital of Soochow University, Soochow, China \\ 2 Department of Critical Care Medicine, Wuxi People's Hospital affiliated to Nanjing Medical University, Wuxi, China \\ 3 Department of Emergency Medicine, Affiliated Hospital of Jiangnan University, Wuxi, China \\ 4 Department of Endocrinology and Metabolism, Wuxi People's Hospital affiliated to Nanjing Medical University, Wuxi, China \\ Department of Critical Care Medicine, Taizhou Central Hospital Taizhou University, Taizhou, China \\ Corresponding Authors: Lijun Liu, Liang Dong \\ Email address: liulijun@suda.edu.cn, dongliangphd@gmail.com
}

Objective: To investigate serum intestinal fatty acid-binding protein (I-FABP) in two groups of patients with different duration of hyperglycemia in a cross-sectional study.

Materials and methods: In the present study, a total of 280 individuals (158 outpatients and 122 inpatients) suffering from hyperglycemia were recruited between May and September 2019. The clinical information of all participants was collected from the hospital information system, including the duration of hyperglycemia, age, gender, hemoglobin Alc ( $\mathrm{HbAlc}), 75-\mathrm{g}$ oral glucose tolerance test including fasting plasma glucose (FPG), 2-hour plasma glucose (2hPG), fasting C-peptide (FC-pep), 2-hour Cpeptide (2hC-pep), fasting insulin (FIns), and 2-hour insulin ( $2 \mathrm{hlns}$ ). In addition, the morbidity of diabetic complications (retinopathy, neuropathy, and nephropathy) in the inpatient group was determined. Furthermore, the difference between $2 \mathrm{hPG}$ and FPG $(\Delta \mathrm{PG})$, the difference between $2 \mathrm{hC}$-pep and FC-pep $(\Delta \mathrm{C}$-pep), and the difference between $2 \mathrm{hlns}$ and FIns ( $\Delta \mathrm{Ins})$ were calculated. The level of serum I-FABP, a biomarker of intestinal barrier (IB) dysfunction, was estimated by an enzyme-linked immunosorbent assay.

Results: For the outpatient group, the median duration of hyperglycemia was less than a year; the serum I-FABP level was positively correlated with age $(R=0.299, P<0.001)$. For the inpatient group, the median duration of hyperglycemia was ten years; correlation analysis showed that the serum I-FABP level was positively associated with age and $\triangle P G(R=0.286, P=0.001 ; R=0.250, P=0.006$, respectively) while negatively associated with FC-pep and 2hC-pep $(R=-0.304, P=0.001 ; R=-0.241, P=0.008$, respectively); multiple linear regression analysis showed that the serum I-FABP level was positively associated with the duration of hyperglycemia $(\beta=0.362, P<0.001)$; moreover, patients with retinopathy had a significantly higher I-FABP level than those without retinopathy $(P=0.001)$.

Conclusions: In the outpatients whose duration of hyperglycemia was less than a year, the serum IFABP level was positively associated with age. In the inpatients with different courses of diabetes, the serum I-FABP level was positively associated with the duration of hyperglycemia and glycemic variability but negatively associated with islet beta-cell function; moreover, the serum I-FABP level was higher in patients with retinopathy than in those without retinopathy, suggesting that the IB dysfunction got worse with the progression of diabetes. 


\section{Intestinal fatty acid-binding protein, a biomarker of}

2 intestinal barrier dysfunction, increases with the progression

\section{of type 2 diabetes}

5 Yifeng Wang ${ }^{1,2}$, Licheng Ding ${ }^{3}$, Jiayue Yang ${ }^{4}$, Lijun Liu ${ }^{1}$, Liang Dong ${ }^{5}$

$6{ }^{1}$ Department of Emergency and Critical Care Medicine, Second Affiliated Hospital of Soochow

7 University, No. 1055 Sanxiang Road, Soochow, 215004, Jiangsu, China

$8 \quad 2$ Department of Critical Care Medicine, Wuxi People's Hospital affiliated to Nanjing Medical

9 University, No. 299 Qingyang Road, Wuxi, 214023, Jiangsu, China

$10{ }^{3}$ Department of Emergency Medicine, Affiliated Hospital of Jiangnan University, No. 200 Huihe

11 Road, Wuxi, 214062, Jiangsu, China

$12{ }^{4}$ Department of Endocrinology and Metabolism, Wuxi People's Hospital affiliated to Nanjing

13 Medical University, No. 299 Qingyang Road, Wuxi, 214023, Jiangsu, China

$14{ }^{5}$ Department of Critical Care Medicine, Taizhou Central Hospital affiliated to Taizhou University,

15 No. 999 Donghai Avenue, Taizhou, 318000, Zhejiang, China

17 Corresponding authors:

18 (1) Lijun Liu

19 Department of Emergency and Critical Care Medicine, Second Affiliated Hospital of Soochow

20 University, No. 1055 Sanxiang Road, Soochow, 215004, Jiangsu, China

21 E-mail: liulijun@suda.edu.cn 
22 Contact number: +8613962192756

23

24 (2) Liang Dong

25 Department of Critical Care Medicine, Taizhou Central Hospital affiliated to Taizhou University,

26 No. 999 Donghai Avenue, Taizhou, 318000, Zhejiang, China

27 E-mail: dongliangphd@gmail.com

28 Contact number: +8613812281996 
31 Abstract

Objective: To investigate serum intestinal fatty acid-binding protein (I-FABP) in two groups of patients with different duration of hyperglycemia in a cross-sectional study.

Materials and methods: In the present study, a total of 280 individuals (158 outpatients and 122 inpatients) suffering from hyperglycemia were recruited between May and September 2019. The clinical information of all participants was collected from the hospital information system, including the duration of hyperglycemia, age, gender, hemoglobin A1c (HbA1c), 75-g oral glucose tolerance test including fasting plasma glucose (FPG), 2-hour plasma glucose (2hPG), fasting Cpeptide (FC-pep), 2-hour C-peptide (2hC-pep), fasting insulin (FIns), and 2-hour insulin (2hIns).

In addition, the morbidity of diabetic complications (retinopathy, neuropathy, and nephropathy) in the inpatient group was determined. Furthermore, the difference between $2 \mathrm{hPG}$ and FPG $(\Delta \mathrm{PG})$, the difference between 2hC-pep and FC-pep ( $\Delta \mathrm{C}$-pep), and the difference between $2 \mathrm{hIns}$ and FIns ( $\triangle$ Ins) were calculated. The level of serum I-FABP, a biomarker of intestinal barrier (IB) dysfunction, was estimated by an enzyme-linked immunosorbent assay.

Results: For the outpatient group, the median duration of hyperglycemia was less than a year; the serum I-FABP level was positively correlated with age $(\mathrm{R}=0.299, \mathrm{P}<0.001)$. For the inpatient group, the median duration of hyperglycemia was ten years; correlation analysis showed that the serum I-FABP level was positively associated with age and $\triangle \mathrm{PG}(\mathrm{R}=0.286, \mathrm{P}=0.001 ; \mathrm{R}=0.250$, $\mathrm{P}=0.006$, respectively) while negatively associated with FC-pep and $2 \mathrm{hC}$-pep $(\mathrm{R}=-0.304, \mathrm{P}=$ 
$520.001 ; \mathrm{R}=-0.241, \mathrm{P}=0.008$, respectively); multiple linear regression analysis showed that the 53 serum I-FABP level was positively associated with the duration of hyperglycemia $(\beta=0.362, \mathrm{P}<$ 54 0.001); moreover, patients with retinopathy had a significantly higher I-FABP level than those 55 without retinopathy $(\mathrm{P}=0.001)$.

57 Conclusions: In the outpatients whose duration of hyperglycemia was less than a year, the serum

58 I-FABP level was positively associated with age. In the inpatients with different courses of 59 diabetes, the serum I-FABP level was positively associated with the duration of hyperglycemia 60 and glycemic variability but negatively associated with islet beta-cell function; moreover, the 61 serum I-FABP level was higher in patients with retinopathy than in those without retinopathy, 62 suggesting that the IB dysfunction got worse with the progression of diabetes. 
64 Keywords: Intestinal fatty acid-binding protein (I-FABP), Intestinal barrier (IB) dysfunction, 65 Hyperglycemia, Progression of diabetes 


\section{Introduction}

68

69

Diabetes has become a global public health issue with a growing morbidity and financial burden in the past few decades. Recently, diabetes has been considered as a risk factor of a worse prognosis in patients with COVID-19 ${ }^{1}$. Interestingly, as the most typical clinical feature of diabetes, hyperglycemia has been confirmed to be an essential predictor of adverse outcomes in diabetic as well as in nondiabetic patients in a previous study ${ }^{2}$. The association between hyperglycemia and adverse outcomes in nondiabetic patients is apparent because reversible hyperglycemia, also known as stress-induced hyperglycemia $(\mathrm{SIH})$, is a secondary symptom of primary disease and a well-known marker of disease severity ${ }^{3}$ However, since hyperglycemia exists long before other diseases appear in diabetic patients, the relationship between preexisting hyperglycemia and adverse outcomes needs to be investigated.

Adverse outcomes associated with diabetes can partly be explained by the vulnerablity of diabetic patients to systemic infection and inflammatory response ${ }^{4}$. Nowadays, intestinal barrier (IB) dysfunction induced by hyperglycemia ${ }^{5}$ is considered to be the underlying mechanism of systemic infection and inflammatory response in diabetic patients. The IB is the interface between the gut microbiome and the human body, it prevents the gut microbiome and other deleterious intestinal contents from crossing the barrier during enteral nutrition absorption. In addition, IB dysfunction results in the translocation of intestinal contents, which could be the direct cause of systemic infection and inflammatory response ${ }^{6}$. In a study by Thaiss, hyperglycemia was confirmed to be an independent risk factor for IB dysfunction in animals; furthermore, the association between 
hyperglycemia and IB dysfunction was observed to be time-dependent and dose-dependent in vitro ${ }^{5}$. Although the relationship between hyperglycemia and IB dysfunction has been clarified in animals and cell-based models ${ }^{5}$, the clinical evidence in humans is lacking.

In this study, the serum concentration of intestinal fatty acid-binding protein (I-FABP) was used to indicate the severity of IB dysfunction. I-FABP is an intracellular protein specifically and abundantly expressed in intestinal epithelial cells, and its increased serum concentration represents intestinal epithelial cell damage and IB dysfunction ${ }^{7,8}$. As a biomarker of IB dysfunction, I-FABP determination has been used in patients with necrotizing enterocolitis ${ }^{9}$, acute mesenteric ischemia $^{10}$, strangulated small bowel obstruction ${ }^{11}$, Crohn's disease ${ }^{12}$, blunt trauma ${ }^{13}$, celiac disease $^{14}$, acute pancreatitis ${ }^{15,16}$, acute decompensated heart failure ${ }^{17}$, chronic renal failure ${ }^{18}$, septic shock $^{19}$, psoriasis ${ }^{20}$, and even physiological stressor-induced intestinal damage ${ }^{21}$. In addition to serum I-FABP, many other biomarkers are used to measure IB function ${ }^{8,22}$. However, in this study, the serum I-FABP level was determined to evaluate IB dysfunction as it is convenient to measure in a noninvasive manner.

Diabetic and prediabetic patients with different severities and durations of hyperglycemia were recruited, and their serum I-FABP levels were measured in this study.

07 Materials and methods

\section{Study participants}


109

110

111 Nanjing Medical University. The outpatients were included in this study if they had been tested to

112 be hyperglycemic or had diabetes-related symptoms within a year without taking antidiabetic

113 drugs. The inpatients were included in this study if they had been diagnosed with diabetes and

114 were newly admitted to the inpatient ward. However, participants were excluded from this study

115 if they were younger than 18 years old; had SIH, type 1 diabetes, or a change in lifestyle (e.g., diet

116

117

118

119

120

121

122

123

124

125

126

127

128

129

In this cross-sectional study, participants were recruited from the outpatient clinic and the inpatient ward of the Department of Endocrinology and Metabolism, Wuxi People's Hospital affiliated to

or exercise) in the past year; were pregnant in the past year; or had acute complications of diabetes,

severe hepatic, renal, or heart insufficiency, acute digestive system disease, or abdominal surgery

in the past half year or acute infection in the past month. All patients who agreed to this study and

met the inclusion criteria were included in the analysis. Most conditions causing an increase in the

serum I-FABP level were excluded according to the criteria, which might have caused a selection

bias. Patients who suffered from complications of severe nephropathy were not included in this

study. They were admitted to the Nephrology Department rather than the Department of Endocrinology and Metabolism, which resulted in a selection bias.

25 The present study was approved by the Research Ethics Committee of Wuxi People's Hospital affiliated to Nanjing Medical University (HS2019003). Each participant signed an informed consent form. This study was registered at the Chinese Clinical Trial Register Center (ChiCTR1900022026) and was carried out from May to September 2019. All data can be obtained from the corresponding authors on demand. 


\section{Clinical information}

132 The clinical information of all participants was collected from the hospital information system,

133 including the duration of hyperglycemia, age, gender, hemoglobin A1c (HbA1c), 75-g oral glucose

134 tolerance test including fasting plasma glucose (FPG), 2-hour plasma glucose (2hPG), fasting C-

135 peptide (FC-pep), 2-hour C-peptide (2hC-pep), fasting insulin (FIns), and 2-hour insulin (2hIns).

136 In addition, the morbidity of diabetic complications (retinopathy, neuropathy, and nephropathy) in

137 the inpatient group was determined. Furthermore, the difference between $2 \mathrm{hPG}$ and FPG $(\Delta \mathrm{PG})$,

138 the difference between 2hC-pep and FC-pep ( $\Delta \mathrm{C}$-pep), and the difference between $2 \mathrm{hIns}$ and FIns

139 ( $\Delta$ Ins) were calculated.

\section{Serum I-FABP estimation}

142 All of the fasting blood samples collected from the patients to perform the necessary tests were stored at $2-8{ }^{\circ} \mathrm{C}$ in the clinical laboratory of Wuxi People's Hospital. Then certain serum samples

144 of the enrolled patients were aliquoted and stored at $-80^{\circ} \mathrm{C}$ within 8 hours for future research. The serum concentration of I-FABP was estimated in duplicate by enzyme-linked immunosorbent assay (R\&D, USA and Canada, Catalog Number DFBP20) according to the standardized protocol, and the estimated mean values were used for further analysis. 
150 Statistical analysis was assessed using SPSS 25 (IBM Corporation, Chicago, IL, USA).

151 Continuous variables were assessed for normality using the Kolmogorov-Smirnov test. Variables

152 with a non-normal distribution were summarized by the median and interquartile range.

153 Differences between two groups of continuous variables with non-normal distributions were

154 assessed by the Mann-Whitney test. Differences between categorical variables were assessed by

155 the chi-squared test. The relationships between two continuous variables were assessed by the

156 Spearman correlation coefficient. Logarithmic transformation was performed when necessarily.

157 The associations showing a P-value $<0.05$ in the correlation analysis were included in the

158 multiple linear regression analysis. Only those metrics with a P-value $<0.05$ were kept in the

159 final multiple linear regression models with stepwise variable selection. Finally, all of the

160 differences were considered significant at a 5\% level.

161

162 Results

163 Serum I-FABP in the outpatient group

164 The outpatients were divided into prediabetes group and diabetes group according to the latest 165 diagnosis standards for diabetes ${ }^{23}$. The clinical information and serum I-FABP levels of these two 166 groups are tabulated in Table 1. Compared with the prediabetes group, the diabetes group had a higher HbA1c, FPG, FC-pep, 2hPG, and $\Delta$ PG but a lower 2hC-pep, 2hIns, $\Delta$ C-pep, and $\Delta$ Ins. However, there were no statistically significant differences in age, FIns, or serum I-FABP between these two groups. 
171 Furthermore, the correlation analysis of the serum I-FABP level was compared with age, HbA1c,

172 FPG, FC-pep, FIns, 2hPG, 2hC-pep, 2hIns, $\Delta$ PG, $\Delta$ C-pep, and $\Delta$ Ins. However, age was the only

173 metric that was statistically correlated with the serum I-FABP level in the outpatient group (Table

174 2).

175

176

\section{Serum I-FABP in the inpatient group}

177

The correlation analysis for the inpatient group showed that the serum I-FABP level was significantly correlated with age, duration of hyperglycemia, FC-pep, 2hC-pep, and $\triangle \mathrm{PG}$ but not significantly correlated with the other metrics. However, the duration of hyperglycemia was the only metric that was statistically associated with the serum I-FABP level in the multiple linear regression analysis (Table 3 ).

Finally, we investigated the relationship between the serum I-FABP level and diabetic complications (retinopathy, neuropathy, and nephropathy). The inpatients were divided into complication-positive group and complication-negative group, and the differences of the serum I-

FABP level between the complication-positive group and the complication-negative group were estimated. The results showed that the retinopathy-positive group had a higher serum I-FABP level than the retinopathy-negative group, while the differences of the serum I-FABP level for the two other complications were not statistically significant (Table 4).

\section{Serum I-FABP in all participants}


192 The clinical information and serum I-FABP levels of all participants recruited from the outpatient

193 clinic and the inpatient ward are tabulated in Table 5. The median duration of hyperglycemia was

194 less than a year for the outpatients, while it was ten years for the inpatients. Moreover, compared

195 with the outpatients, the inpatients had higher age, HbAlc, FPG, 2hPG, $\triangle$ PG, and I-FABP but

196 lower FC-pep, FIns, 2hC-pep, 2hIns, $\Delta$ C-pep, and $\Delta$ Ins.

197

198

199

200

201

202

203

204

205

206

207

208

209

210

211

212

Next, correlation analysis of the serum I-FABP level showed a statistical significance with age, duration of hyperglycemia, HbAlc, FC-pep, 2hPG, 2hC-pep, $\Delta \mathrm{PG}$, and $\Delta \mathrm{C}$-pep. Furthermore, multivariate linear regression analysis showed that the serum I-FABP level was positively associated with the age and the duration of hyperglycemia in all participants (Table 6).

\section{Discussion}

A previous study has confirmed the relationship between hyperglycemia and IB dysfunction in animals and cell-based models ${ }^{5}$, and our study added evidence from humans to support this relationship. In the present study, we investigated the serum I-FABP levels in diabetic and prediabetic patients who suffered from different severities and durations of hyperglycemia. Our results showed that in the outpatients whose duration of hyperglycemia was less than a year, the serum I-FABP level was positively associated with age. In the inpatients with different courses of diabetes, the serum I-FABP level was positively associated with the duration of hyperglycemia and glycemic variability but negatively associated with islet beta-cell function; moreover, the serum I-FABP level was higher in patients with retinopathy than in those without retinopathy, 
213 suggesting that the IB dysfunction got worse with the progression of diabetes.

214

215

IB dysfunction and severity of hyperglycemia: the serum I-FABP level was not associated

with FPG, 2hPG, or HbA1c in outpatients.

217 The study by Thaiss confirmed the dose-dependent relationship between IB dysfunction and

hyperglycemia in vitro ${ }^{5}$. Since hyperglycemia in the outpatient group was just identified within a

219 year and untreated, this group was suitable for investigation of the relationship between

hyperglycemia severity and IB dysfunction. FPG and 2hPG show instantaneous plasma glucose

(PG) levels, while HbA1c shows the average PG level in the past 2-3 months ${ }^{23}$. These three metrics

were used to determine the severity of hyperglycemia in this study. Unfortunately, none of them

showed a statistical correlation with the serum I-FABP level in the outpatient group, and

correlation analysis of the inpatient group showed similar results.

These negative results might be explained by the lower severity of hyperglycemia in our clinical

study compared with the in vitro study by Thaiss. In their in vitro study, IB dysfunction was

observed at a glucose level of $8 \mathrm{~g} / \mathrm{L}$ (about $44 \mathrm{mmol} / \mathrm{L})^{5}$, which is rarely achieved in clinical

patients. In other words, the hyperglycemia in our study was not severe enough to cause a statistical

difference in the serum I-FABP level. Therefore, a well-designed study in humans is needed in the

future to answer the dose-dependent relationship between IB dysfunction and hyperglycemia. 
234

235

236

237

238

239

240

241

242

244

245

246

247

248

249

250

251

252

253

254

\section{the duration of hyperglycemia in inpatients.}

After dose-dependent relationship discussed above, time-dependent relationship was assessed, the relationship between IB dysfunction and the duration of hyperglycemia was investigated in the study. The median duration of hyperglycemia was less than a year for the outpatient group, while it was ten years for the inpatient group. For the inpatient group, the serum I-FABP level was statistically associated with the duration of hyperglycemia both according to the correlation analysis and the multivariate linear regression analysis.

The relationship between IB dysfunction and the duration of hyperglycemia can be explained by the accumulation of advanced glycation end products (AGEs) caused by the long-standing hyperglycemic state in diabetes. AGEs have been demonstrated to contribute to diabetic complications in many studies, and the accumulation of AGEs and the activation of their receptors (RAGE) induce NADPH oxidase stimulation, reactive oxygen intermediate formation, nuclear factor- $\kappa \mathrm{B}$ activation, and gene transcription, which further lead to a sustained inflammatory reaction, cell damage, and organ dysfunction ${ }^{24}$. Surprisingly, both the accumulation of AGEs and the overexpression of RAGE were observed in the gastrointestinal tract of diabetic rats ${ }^{25}$, which contributes to IB dysfunction in diabetes.

IB dysfunction and progression of diabetes: the serum I-FABP level was associated with retinopathy, glycemic variability and islet beta-cell dysfunction in inpatients.

As a result of sustained hyperglycemia, diabetic complications develop with the progression of 
255 diabetes. In this study, the serum I-FABP level was higher in patients with retinopathy than in

256 those without retinopathy in the inpatient group. As retinopathy is a complication that develops

257 with the progression of diabetes, this result suggested that the IB dysfunction got worse with the 258 progression of diabetes. Unfortunately, patients who suffered from severe nephropathy were not 259 included in this study, this partly explained why the serum I-FABP level was not higher in the patients with neuropathy or nephropathy.

Previous studies have found that glycemic variability is closely related to diabetic complications.

Glycemic variability consists of the magnitude of PG excursions and the frequency of the fluctuations. Compared with sustained hyperglycemia, glycemic variability has more deleterious effects in the pathogenesis of diabetic complications ${ }^{26,27}$. An observational study has found that type 2 diabetic patients incapable of maintaining stable PG levels were more likely to have IB injury $^{28}$, in other words, glycemic variability might be associated with IB dysfunction. In this study, $\triangle \mathrm{PG}$ was used to indicate the glycemic variability, and the results showed that the serum IFABP level was positively correlated with $\triangle \mathrm{PG}$ in the inpatient group. Taken together, IB dysfunction was related to glycemic variability and was aggravated with the progression of diabetes. Since $\triangle \mathrm{PG}$ is not enough to show the whole day glycemic variability, better designed studies are needed to further estimate the relationship between glycemic variability and IB dysfunction.

In addition, islet beta-cell dysfunction advances with the progression of diabetes ${ }^{29}$. In this study, 
276 the serum C-pep and insulin levels were used to indicate islet beta-cell function. The results

277 showed that the serum I-FABP level was negatively associated with the C-pep (FC-pep and 2hC-

278 pep) levels in the inpatient group. However, there was no statistical correlation between the serum

279 I-FABP and insulin (FIns and 2hIns) levels. C-pep is secreted from beta cells at an equimolar

concentration as insulin, however, the half-life of C-pep is longer than that of insulin, ${ }^{30}$ which

makes C-pep a better metric for islet beta-cell function. In this study, the investigation of C-pep

suggested that the aggravation of IB dysfunction was accompanied by the loss of islet beta-cell

function in the progression of diabetes.

Although the serum I-FABP level was correlated with $\triangle \mathrm{PG}$, FC-pep, and 2hC-pep, these

associations disappeared in the multivariate linear regression analysis. Given the roles of diabetic

complications, glycemic variability, and advancing islet beta-cell dysfunction in the progression

of diabetes, $\triangle \mathrm{PG}$, FC-pep, and 2hC-pep were considered to be confounding factors in the

relationship between the serum I-FABP level and the duration of hyperglycemia. Since the

duration of hyperglycemia closely followed the course of diabetes, this study suggested that the

IB dysfunction got worse with the progression of diabetes.

IB dysfunction and age: the serum I-FABP level was positively correlated with age in

\section{outpatients and inpatients.}

In the outpatient group, without the influence of the duration of hyperglycemia, the serum I-FABP

level was positively correlated with age, suggesting that the older patients suffered from more 
297

severe IB dysfunction in the newly diagnosed diabetic and prediabetic patients. A previous study has found that IB dysfunction might be an important event in the aging process, and that is conserved across a broad range of species ${ }^{31}$. Age-related loss of the heat-shock transcription factor has been confirmed to be involved in IB dysfunction by accelerating the decay of the intestinal subapical terminal web and impairing its interactions with cell junctions in C. elegans ${ }^{32}$. The current study added evidence regarding the association between IB dysfunction and age in humans.

Similarly, in the inpatient group, the serum I-FABP level was positively correlated with age. However, the association between I-FABP and age disappeared in the multivariate linear regression analysis, leaving the duration of hyperglycemia as the only metric associated with the serum I-FABP level. Considering that the durations of hyperglycemia get longer when diabetic patients grow older, age might be a confounding factor in the relationship between the serum IFABP level and the duration of hyperglycemia in the inpatient group.

\section{The different behaviors of two groups}

At first glance, the results in all participants seem to be the combination of two groups: correlation analysis showed the serum I-FABP level was positively associated with the duration of hyperglycemia, age, and glycemic variability but negatively associated with islet beta-cell function; multivariate linear regression analysis showed that the serum I-FABP level was associated with both age and the duration of hyperglycemia. 
318 When all patients were combined, there was some evidence of a relationship, but on further

319 investigation, it turned out that there appeared to be different behaviors in each group, and the

320 difference might be mainly caused by the significant difference in the duration of hyperglycemia.

321 As a result, the relationship between I-FAPB and the other metrics reported for all participants

322 might be driven by the group sizes. Consequently, the results for all participants need to be

323 interpreted with caution.

324

325

Moreover, in all participants and in the inpatient group, the multivariate linear regression models

were just ways of determining possible associations between I-FABP and the metrics, the direct causal relationship in humans needs further investigation.

\section{Perspective of the study}

Based on the results of this study, we speculated that IB dysfunction occurred and developed along with the progression of diabetes. The relationship between hyperglycemia and IB dysfunction offers a new perspective on the clinical phenomenon of diabetes. On one hand, an impaired IB enhances the influx and systemic dissemination of intestinal bacteria, which explains the vulnerability of diabetic patients to infection ${ }^{33}$. On the other hand, more intestinal contents and bacterial metabolites come across the impaired IB and are released into the blood circulation, causing systemic chronic oxidative stress and inflammatory response, which not only promote diabetic complications ${ }^{34,35}$ but also contribute directly to diabetes ${ }^{36}$. 
339 The association between the duration of hyperglycemia and IB dysfunction was clarified in this

340 study, but the underlying mechanisms have not been discussed. Previous studies showed that

341 nondiabetic patients who suffered from SIH were more likely to get diabetes in the future ${ }^{37,38}$,

342 which brought about a confusing hypothesis that reversible hyperglycemia could cause irreversible

343 hyperglycemia. It is well recognized that some primary diseases like sepsis, trauma, burns, and

344 surgery, which induce $\mathrm{SIH}^{3}$, can directly cause IB dysfunction ${ }^{39}$. Hyperglycemia-induced IB

345 dysfunction allows for an enhanced influx of intestinal contents, and the induced infection and

346 inflammatory response further intensify hyperglycemia ${ }^{3}$. Surprisingly, IB dysfunction builds a

347 bridge between SIH and diabetes, that is, IB dysfunction not only results from but also contributes

348 to hyperglycemia. Since improving IB function by treating the primary disease has been shown to

349 alleviate $\mathrm{SIH}^{3}$, aiming to improve IB function might be a promising treatment strategy for diabetes.

Besides the new therapeutic approach for hyperglycemia, some widely used antidiabetic drugs

have already shown protective potential for IB. Glucagon-like peptide 1, which controls meal-

related glycemic excursions, alleviates gut inflammation and promotes the repairment of intestinal

epithelial cells ${ }^{40,41}$. Similar protective effects have also been observed with metformin ${ }^{42}$ and

berberine ${ }^{43}$. Compared with the IB, the gut microbiome has drawn increasing attention in diabetes

research. Although quite a few studies have shown that the gut microbiome influences the 
360 This study had some limitations. First, the participants recruited from the inpatient ward were

361 admitted to the hospital for different pathological conditions, including but not limited to poor

362 glucose control and diabetic complications. The influence of all these conditions on the serum I-

363 FABP level was not thoroughly investigated. Second, the participants recruited from the inpatient

364 ward had been treated with antidiabetic drugs, whether these drugs affected the serum I-FABP

365 level was not investigated. Third, patients who suffered from severe nephropathy complications

366 were not included in this study. Fourth, the results from all participants were driven by the group

367 sizes because of the significant difference in the duration of hyperglycemia between groups.

369 Conclusions

370 To the best of our knowledge, this study investigated the serum I-FABP level in diabetic and

371 prediabetic patients for the first time, and the results showed that in the outpatients whose duration

372 of hyperglycemia was less than a year, the serum I-FABP level was positively associated with age.

373 In the inpatients with different courses of diabetes, the serum I-FABP level was positively

374 associated with the duration of hyperglycemia and glycemic variability but negatively associated

375 with islet beta-cell function; moreover, the serum I-FABP level was higher in patients with retinopathy than in those without retinopathy, suggesting that the IB dysfunction got worse with the progression of diabetes.

Considering the participation of the gut microbiome in the etiology of diabetes, it is difficult to ignore the role of IB, whose dysfunction might cause oxidative stress, systemic infection, and 
381 inflammatory response. By highlighting the IB in diabetes-related research, we offer a new 382 perspective to interpret this familiar disease.

383

\section{Acknowledgements}

385 This manuscript has been edited and proofread by Medjaden Bioscience Limited. 
389 1. Zhu L, She ZG, Cheng X, Qin JJ, Zhang XJ, Cai J, Lei F, Wang H, Xie J, Wang W, Li H, Zhang P, Song

X, Chen X, Xiang M, Zhang C, Bai L, Xiang D, Chen MM, Liu Y, Yan Y, Liu M, Mao W, Zou J, Liu L,

Chen G, Luo P, Xiao B, Zhang C, Zhang Z, Lu Z, Wang J, Lu H, Xia X, Wang D, Liao X, Peng G, Ye P,

Yang J, Yuan Y, Huang X, Guo J, Zhang BH, Li H. Association of Blood Glucose Control and Outcomes

in Patients with COVID-19 and Pre-existing Type 2 Diabetes. Cell Metab. 2020.

Without Diabetes. Journal of diabetes science and technology. 2017;11(6):1272-1273.

3. Mifsud S, Schembri EL, Gruppetta M. Stress-induced hyperglycaemia. British journal of hospital medicine (London, England : 2005). 2018;79(11):634-639.

4. Knapp S. Diabetes and infection: is there a link?--A mini-review. Gerontology. 2013;59(2):99-104.

5. Thaiss CA, Levy M, Grosheva I, Zheng D, Soffer E, Blacher E, Braverman S, Tengeler AC, Barak O,

Elazar M, Ben-Zeev R, Lehavi-Regev D, Katz MN, Pevsner-Fischer M, Gertler A, Halpern Z, Harmelin A,

Aamar S, Serradas P, Grosfeld A, Shapiro H, Geiger B, Elinav E. Hyperglycemia drives intestinal barrier dysfunction and risk for enteric infection. Science. 2018;359(6382):1376-1383. intestinal FABPs in the intestine. Prostaglandins, leukotrienes, and essential fatty acids. 2015;93:9-16.

8. Wells JM, Brummer RJ, Derrien M, MacDonald TT, Troost F, Cani PD, Theodorou V, Dekker J, Meheust 
biomarkers. American journal of physiology Gastrointestinal and liver physiology. 2017;312(3):G171-

$4119 . \quad$ Coufal S, Kokesova A, Tlaskalova-Hogenova H, Frybova B, Snajdauf J, Rygl M, Kverka M. Urinary I-

FABP, L-FABP, TFF-3, and SAA Can Diagnose and Predict the Disease Course in Necrotizing

Enterocolitis at the Early Stage of Disease. Journal of immunology research. 2020;2020:3074313.

10. Salim SY, Young PY, Churchill TA, Khadaroo RG. Urine intestinal fatty acid-binding protein predicts acute mesenteric ischemia in patients. The Journal of surgical research. 2017;209:258-265.

11. Kittaka H, Akimoto H, Takeshita H, Funaoka H, Hazui H, Okamoto M, Kobata H, Ohishi Y. Usefulness of intestinal fatty acid-binding protein in predicting strangulated small bowel obstruction. PLoS One. 2014;9(6):e99915.

12. Sarikaya M, Ergul B, Dogan Z, Filik L, Can M, Arslan L. Intestinal fatty acid binding protein (I-FABP) as a promising test for Crohn's disease: a preliminary study. Clinical laboratory. 2015;61(1-2):87-91.

13. Matsumoto S, Sekine K, Funaoka H, Funabiki T, Shimizu M, Hayashida K, Kitano M. Early diagnosis of hollow viscus injury using intestinal fatty acid-binding protein in blunt trauma patients. Medicine (Baltimore). 2017;96(10):e6187.

14. Oldenburger IB, Wolters VM, Kardol-Hoefnagel T, Houwen RHJ, Otten HG. Serum intestinal fatty acidbinding protein in the noninvasive diagnosis of celiac disease. APMIS : acta pathologica, microbiologica, et immunologica Scandinavica. 2018;126(3):186-190.

15. Kupcinskas J, Gedgaudas R, Hartman H, Sippola T, Lindstrom O, Johnson CD, Regner S. Intestinal Fatty Acid Binding Protein as a Marker of Necrosis and Severity in Acute Pancreatitis. Pancreas.

2018;47(6):715-720. 
$430 \quad$ 16. Goswami P, Sonika U, Moka P, Sreenivas V, Saraya A. Intestinal Fatty Acid Binding Protein and Citrulline as Markers of Gut Injury and Prognosis in Patients With Acute Pancreatitis. Pancreas. 2017;46(10):12751280.

17. Kitai T, Kim YH, Kiefer K, Morales R, Borowski AG, Grodin JL, Tang WHW. Circulating intestinal fatty acid-binding protein (I-FABP) levels in acute decompensated heart failure. Clinical biochemistry. 2017;50(9):491-495.

18. Okada K, Sekino M, Funaoka H, Sato S, Ichinomiya T, Murata H, Maekawa T, Nishikido M, Eishi K, Hara research. 2018;230:94-100.

19. Sekino M, Funaoka H, Sato S, Okada K, Inoue H, Yano R, Matsumoto S, Ichinomiya T, Higashijima U, bowel ischemia in patients with septic shock: A preliminary study. J Crit Care. 2017;42:92-100.

20. Sikora M, Stec A, Chrabaszcz M, Waskiel-Burnat A, Zaremba M, Olszewska M, Rudnicka L. Intestinal Fatty Acid Binding Protein, a Biomarker of Intestinal Barrier, is Associated with Severity of Psoriasis. Journal of clinical medicine. 2019;8(7).

21. March DS, Marchbank T, Playford RJ, Jones AW, Thatcher R, Davison G. Intestinal fatty acid-binding protein and gut permeability responses to exercise. Eur J Appl Physiol. 2017;117(5):931-941.

22. Piton G, Capellier G. Biomarkers of gut barrier failure in the ICU. Curr Opin Crit Care. 2016;22(2):152-

23. 2. Classification and Diagnosis of Diabetes: Standards of Medical Care in Diabetes-2020. Diabetes Care. 
451 24. Wautier MP, Guillausseau PJ, Wautier JL. Activation of the receptor for advanced glycation end products and consequences on health. Diabetes \& metabolic syndrome. 2017;11(4):305-309.

25. Chen PM, Gregersen H, Zhao JB. Advanced glycation end-product expression is upregulated in the gastrointestinal tract of type 2 diabetic rats. World journal of diabetes. 2015;6(4):662-672.

26. Beck RW, Bergenstal RM, Riddlesworth TD, Kollman C, Li Z, Brown AS, Close KL. Validation of Time in Range as an Outcome Measure for Diabetes Clinical Trials. Diabetes Care. 2019;42(3):400-405.

27. Nusca A, Tuccinardi D, Albano M, Cavallaro C, Ricottini E, Manfrini S, Pozzilli P, Di Sciascio G. research and reviews. 2018;34(8):e3047.

29. Bergman RN, Finegood DT, Kahn SE. The evolution of beta-cell dysfunction and insulin resistance in type 2 diabetes. Eur J Clin Invest. 2002;32:35-45.

30. Polonsky KS, Rubenstein AH. C-PEPTIDE AS A MEASURE OF THE SECRETION AND HEPATIC EXTRACTION OF INSULIN - PITFALLS AND LIMITATIONS. Diabetes. 1984;33(5):486-494.

31. Dambroise E, Monnier L, Ruisheng L, Aguilaniu H, Joly JS, Tricoire H, Rera M. Two phases of aging separated by the Smurf transition as a public path to death. Scientific reports. $2016 ; 6: 23523$.

32. Egge N, Arneaud SLB, Wales P, Mihelakis M, McClendon J, Fonseca RS, Savelle C, Gonzalez I, Ghorashi A, Yadavalli S, Lehman WJ, Mirzaei H, Douglas PM. Age-Onset Phosphorylation of a Minor Actin Variant Promotes Intestinal Barrier Dysfunction. Developmental cell. 2019;51(5):587-601.e587. 
472 33. Trevelin SC, Carlos D, Beretta M, da Silva JS, Cunha FQ. Diabetes Mellitus and Sepsis: A Challenging Association. Shock (Augusta, Ga). 2017;47(3):276-287.

474
34. Jha JC, Ho F, Dan C, Jandeleit-Dahm K. A causal link between oxidative stress and inflammation in cardiovascular and renal complications of diabetes. Clinical science (London, England : 1979).

2018;132(16):1811-1836.

35. Volpe CMO, Villar-Delfino PH, Dos Anjos PMF, Nogueira-Machado JA. Cellular death, reactive oxygen species (ROS) and diabetic complications. Cell death \& disease. 2018;9(2):119.

36. McCracken E, Monaghan M, Sreenivasan S. Pathophysiology of the metabolic syndrome. Clinics in dermatology. 2018;36(1):14-20.

37. Kar P, Plummer MP, Abdelhamid YA, Giersch EJ, Summers MJ, Weinel LM, Finnis ME, Phillips LK, Jones KL, Horowitz M, Deane AM. Incident Diabetes in Survivors of Critical Illness and Mechanisms Underlying Persistent Glucose Intolerance: A Prospective Cohort Study. Critical Care Medicine. 2019;47(2):E103-E111.

38. Plummer MP, Finnis ME, Phillips LK, Kar P, Bihari S, Biradar V, Moodie S, Horowitz M, Shaw JE, Deane AM. Stress Induced Hyperglycemia and the Subsequent Risk of Type 2 Diabetes in Survivors of Critical Illness. Plos One. 2016;11(11):12.

39. Greis C, Rasuly Z, Janosi RA, Kordelas L, Beelen DW, Liebregts T. Intestinal T lymphocyte homing is associated with gastric emptying and epithelial barrier function in critically ill: a prospective observational study. Critical care (London, England). 2017;21(1):70.

40. Smits MM, van Raalte DH, Tonneijck L, Muskiet MH, Kramer MH, Cahen DL. GLP-1 based therapies: clinical implications for gastroenterologists. Gut. 2016;65(4):702-711. 
493 41. Anbazhagan AN, Thaqi M, Priyamvada S, Jayawardena D, Kumar A, Gujral T, Chatterjee I, Mugarza E, Saksena S, Onyuksel H, Dudeja PK. GLP-1 nanomedicine alleviates gut inflammation. Nanomedicine : nanotechnology, biology, and medicine. 2017;13(2):659-665.

42. Rodriguez J, Hiel S, Delzenne NM. Metformin: old friend, new ways of action-implication of the gut microbiome? Current Opinion in Clinical Nutrition and Metabolic Care. 2018;21(4):294-301.

43. Gong J, Hu M, Huang Z, Fang K, Wang D, Chen Q, Li J, Yang D, Zou X, Xu L, Wang K, Dong H, Lu F. Berberine Attenuates Intestinal Mucosal Barrier Dysfunction in Type 2 Diabetic Rats. Frontiers in pharmacology. 2017;8:42.

44. Hills RD, Pontefract BA, Mishcon HR, Black CA, Sutton SC, Theberge CR. Gut Microbiome: Profound Implications for Diet and Disease. Nutrients. 2019;11(7):40.

45. Canfora EE, Meex RCR, Venema K, Blaak EE. Gut microbial metabolites in obesity, NAFLD and T2DM. Nature Reviews Endocrinology. 2019;15(5):261-273. 


\section{Abbreviation:}

508 I-FABP: intestinal fatty acid-binding protein

509 IB: intestinal barrier

$510 \quad$ HbA1c: hemoglobin A1c

511 OGTT: oral glucose tolerance test

512 PG: plasma glucose

513 C-pep: C-peptide

$514 \quad$ Ins: insulin

$515 \quad$ FPG: fasting plasma glucose

$516 \quad$ FC-pep: fasting C-peptide

FIns: fasting insulin

2hPG: 2-hour plasma glucose

519

2hC-pep: 2-hour C-peptide

520

2hIns: 2-hour insulin

$\triangle \mathrm{PG}$ : the difference between $2 \mathrm{hPG}$ and $\mathrm{FPG}$

$\Delta \mathrm{C}$-pep: the difference between 2hC-pep and FC-pep

$\Delta$ Ins: the difference between $2 \mathrm{hIns}$ and FIns

SIH: stress-induced hyperglycemia

AGEs: advanced glycation end products

RAGE: AGEs receptor 


\section{Table 1 (on next page)}

Clinical information and the serum I-FABP level between the different stages of diabetes for the outpatient group. 
1 Table 1. Clinical information and the serum I-FABP level between the different stages of diabetes 2 for the outpatient group.

\begin{tabular}{|c|c|c|c|}
\hline & Prediabetes & Diabetes & P-value \\
\hline Male/Female, $\mathrm{n} * *$ & $42 / 53$ & $48 / 15$ & $<0.001$ \\
\hline Age, $y$ & $45(32,57)$ & $49(34,55)$ & 0.790 \\
\hline HbA1c, $\% * *$ & $5.5(5.3,5.8)$ & $6.9(6.4,9.1)$ & $<0.001$ \\
\hline $\mathrm{FPG}, \mathrm{mmol} / \mathrm{L} * *$ & $5.94(5.41,6.47)$ & $8.54(7.64,10.89)$ & $<0.001$ \\
\hline FC-pep, ng/mL ** & $2.39(1.86,3.16)$ & $2.97(2.29,3.62)$ & 0.004 \\
\hline FIns, mu/L & $10.21(5.91,14.36)$ & $11.98(7.37,15.13)$ & 0.136 \\
\hline $2 \mathrm{hPG}, \mathrm{mmol} / \mathrm{L} * *$ & $8.46(6.93,10.28)$ & $15.73(13.55,19.25)$ & $<0.001$ \\
\hline 2hC-pep, ng/mL ** & $9.96(7.61,12.83)$ & $7.35(5.89,11.15)$ & 0.001 \\
\hline $2 \mathrm{hIns}, \mathrm{mu} / \mathrm{L} * *$ & $64.26(42.18,122.03)$ & $46.56(28.78,69.34)$ & 0.003 \\
\hline$\Delta \mathrm{PG}, \mathrm{mmol} / \mathrm{L} * *$ & $2.52(1.00,4.00)$ & $7.28(4.81,9.21)$ & $<0.001$ \\
\hline$\Delta \mathrm{C}$-pep, ng/mL $* *$ & $7.56(5.57,10.36)$ & $4.63(3.03,7.96)$ & $<0.001$ \\
\hline$\Delta \mathrm{Ins}, \mathrm{mu} / \mathrm{L} * *$ & $57.25(33.78,105.98)$ & $34.82(18.20,58.76)$ & $<0.001$ \\
\hline I-FABP, pg/mL & $1354(948,1634)$ & $1231(883,1604)$ & 0.442 \\
\hline
\end{tabular}

$3 \mathrm{I}-\mathrm{FABP}=$ intestinal fatty acid-binding protein, $\mathrm{HbA1c}=$ hemoglobin $\mathrm{A} 1 \mathrm{c}, \mathrm{FPG}=$ fasting plasma

4 glucose, FC-pep = fasting C-peptide, FIns = fasting insulin, 2hPG = 2-hour plasma glucose, $2 \mathrm{hC}$ -

5 pep $=2$-hour C-peptide, 2 hIns $=2$-hour insulin, $\Delta \mathrm{PG}=2 \mathrm{hPG}-\mathrm{FPG}, \Delta \mathrm{C}$-pep $=2 \mathrm{hC}$-pep $-\mathrm{FC}$ -

6 pep, $\Delta \operatorname{Ins}=2 \mathrm{hIns}-$ FIns.

$7 \quad * * \mathrm{P}<0.01$. 
Table 2 (on next page)

Correlation analysis of the serum I-FABP level for the outpatient group. 
1 Table 2. Correlation analysis of the serum I-FABP level for the outpatient group.

\begin{tabular}{|c|c|c|c|}
\hline & & $\mathrm{R}$ & P-value \\
\hline \multirow[t]{11}{*}{ I-FABP } & Age ** & 0.299 & $<0.001$ \\
\hline & HbA1c & -0.027 & 0.734 \\
\hline & FPG & -0.052 & 0.515 \\
\hline & FC-pep & 0.031 & 0.696 \\
\hline & FIns & 0.010 & 0.899 \\
\hline & $2 \mathrm{hPG}$ & -0.025 & 0.757 \\
\hline & 2hC-pep & 0.104 & 0.197 \\
\hline & $2 \mathrm{hIns}$ & 0.063 & 0.435 \\
\hline & $\Delta \mathrm{PG}$ & 0.003 & 0.973 \\
\hline & $\Delta \mathrm{C}$-pep & 0.103 & 0.199 \\
\hline & $\Delta \mathrm{Ins}$ & 0.080 & 0.322 \\
\hline
\end{tabular}

$2 \mathrm{I}-\mathrm{FABP}=$ intestinal fatty acid-binding protein, $\mathrm{HbA1c}=$ hemoglobin $\mathrm{A} 1 \mathrm{c}, \mathrm{FPG}=$ fasting plasma

3 glucose, FC-pep = fasting C-peptide, FIns = fasting insulin, $2 \mathrm{hPG}=2$-hour plasma glucose, $2 \mathrm{hC}$ -

4 pep = 2-hour C-peptide, 2hIns = 2-hour insulin, $\Delta \mathrm{PG}=2 \mathrm{hPG}-\mathrm{FPG}, \Delta \mathrm{C}$-pep $=2 \mathrm{hC}$-pep $-\mathrm{FC}$ -

5 pep, $\Delta \operatorname{Ins}=2 \mathrm{hIns}-$ FIns.

$6 * * \mathrm{P}<0.01$. 


\section{Table 3(on next page)}

Correlation analysis and multiple linear regression analysis of the serum I-FABP level for the inpatient group. 
1 Table 3. Correlation analysis and multiple linear regression analysis of the serum I-FABP level for 2 the inpatient group.

\begin{tabular}{|c|c|c|c|c|}
\hline & \multicolumn{2}{|c|}{ Correlation Analysis } & \multicolumn{2}{|c|}{ Multiple Linear Regression } \\
\hline & $\mathrm{R}$ & P-value & $\beta$ & P-value \\
\hline Age & 0.286 & 0.001 & 0.098 & 0.317 \\
\hline Duration & 0.350 & $<0.001$ & 0.362 & $<0.001$ \\
\hline HbA1c & 0.045 & 0.625 & - & - \\
\hline FPG & -0.126 & 0.168 & - & - \\
\hline FC-pep & -0.304 & 0.001 & -0.090 & 0.352 \\
\hline FIns & -0.145 & 0.244 & - & - \\
\hline $2 \mathrm{hPG}$ & 0.149 & 0.105 & - & - \\
\hline 2hC-pep & -0.241 & 0.008 & -0.064 & 0.494 \\
\hline $2 \mathrm{hIns}$ & -0.219 & 0.081 & - & - \\
\hline$\Delta \mathrm{PG}$ & 0.250 & 0.006 & 0.149 & 0.110 \\
\hline$\Delta \mathrm{C}$-pep & -0.163 & 0.076 & - & - \\
\hline$\Delta \operatorname{Ins}$ & -0.209 & 0.097 & - & - \\
\hline
\end{tabular}

3 The corresponding model was $\mathrm{Y}=7.292+0.027^{*} \mathrm{X}, \mathrm{Y}: \ln (\mathrm{I}-\mathrm{FABP}), \mathrm{X}$ : Duration, and $\mathrm{R}^{2}=0.131$ 4 in the multiple linear regression. I-FABP = intestinal fatty acid-binding protein, HbA1c = 5 hemoglobin A1c, FPG = fasting plasma glucose, FC-pep = fasting C-peptide, FIns = fasting 6 insulin, 2hPG = 2-hour plasma glucose, 2hC-pep = 2-hour C-peptide, 2hIns = 2-hour insulin, $\Delta \mathrm{PG}$ 
$7=2 \mathrm{hPG}-$ FPG,$\Delta \mathrm{C}$-pep $=2 \mathrm{hC}$-pep - FC-pep, $\Delta$ Ins $=2 \mathrm{hIns}-$ FIns. $\beta=$ standardized $\beta$-coefficient. 


\section{Table 4 (on next page)}

Relationship between the serum I-FABP level and diabetic complications for the inpatient group. 
1 Table 4. Relationship between the serum I-FABP level and diabetic complications for the inpatient 2 group.

\begin{tabular}{llccc}
\hline \multirow{2}{*}{ Complications } & Positive & \multicolumn{2}{c}{ I-FABP } & \\
\cline { 3 - 5 } & cases $(\%)$ & Positive & Negative & P-value \\
\hline Retinopathy ** & $48(39.3)$ & $2164(1749,3262)$ & $1585(1168,2467)$ & 0.001 \\
Neuropathy & $51(41.8)$ & $1949(1373,2953)$ & $1697(1169,2617)$ & 0.126 \\
Nephropathy & $34(27.9)$ & $1836(1206,2719)$ & $1829(1256,2638)$ & 0.833 \\
\hline
\end{tabular}

$3 \quad$ I-FABP $=$ intestinal fatty acid-binding protein.

$4 \quad * * \mathrm{P}<0.01$. 


\section{Table 5 (on next page)}

Clinical information and serum I-FABP levels of participants recruited from the outpatient clinic and the inpatient ward. 
1 Table 5. Clinical information and serum I-FABP levels of participants recruited from the outpatient 2 clinic and the inpatient ward.

\begin{tabular}{|c|c|c|c|}
\hline & Outpatient & Inpatient & P-value \\
\hline Male/Female, $\mathrm{n}$ & $90 / 68$ & $72 / 50$ & 0.730 \\
\hline Age, $\mathrm{y} * *$ & $47.5(33,56)$ & $56(51.75,67)$ & $<0.001$ \\
\hline Duration, $\mathrm{y} * *$ & $<1$ & $10(3,14)$ & $<0.001$ \\
\hline HbA1c, $\% * *$ & $5.9(5.5,6.5)$ & $8.0(6.8,9.3)$ & $<0.001$ \\
\hline $\mathrm{FPG}, \mathrm{mmol} / \mathrm{L} *$ & $6.57(5.77,8.04)$ & $7.43(5.86,9.08)$ & 0.037 \\
\hline FC-pep, ng/mL ** & $2.69(1.92,3.45)$ & $1.92(1.29,2.67)$ & $<0.001$ \\
\hline FIns, mu/L * & $10.66(6.29,14.74)$ & $7.77(5.21,12.74)$ & 0.020 \\
\hline $2 \mathrm{hPG}, \mathrm{mmol} / \mathrm{L} * *$ & $10.55(7.87,14.71)$ & $18.97(14.37,21.38)$ & $<0.001$ \\
\hline 2hC-pep, ng/mL ** & $9.22(7.03,12.39)$ & $5.19(3.59,7.69)$ & $<0.001$ \\
\hline $2 \mathrm{hIns}, \mathrm{mu} / \mathrm{L} * *$ & $55.34(35.06,109.25)$ & $38.11(21.67,69.28)$ & 0.001 \\
\hline$\Delta \mathrm{PG}, \mathrm{mmol} / \mathrm{L} * *$ & $3.80(1.91,7.16)$ & $10.32(6.71,12.95)$ & $<0.001$ \\
\hline$\Delta$ C-pep, ng/mL $* *$ & $6.78(4.27,9.36)$ & $3.33(2.13,5.17)$ & $<0.001$ \\
\hline$\Delta \mathrm{Ins}, \mathrm{mu} / \mathrm{L} * *$ & $47.02(26.69,91.97)$ & $30.51(16.84,56.10)$ & 0.002 \\
\hline I-FABP, pg/mL** & $1289(909,1629)$ & $1831(1243,2642)$ & $<0.001$ \\
\hline
\end{tabular}

$3 \mathrm{I}-\mathrm{FABP}=$ intestinal fatty acid-binding protein, $\mathrm{HbA1c}=$ hemoglobin $\mathrm{A} 1 \mathrm{c}, \mathrm{FPG}=$ fasting plasma

4 glucose, FC-pep = fasting C-peptide, FIns = fasting insulin, 2hPG = 2-hour plasma glucose, 2hC5 pep $=2$-hour C-peptide, 2 hIns $=2$-hour insulin, $\Delta \mathrm{PG}=2 \mathrm{hPG}-\mathrm{FPG}, \Delta \mathrm{C}$-pep $=2 \mathrm{hC}$-pep $-\mathrm{FC}$ 6 pep, $\Delta \operatorname{Ins}=2 \mathrm{hIns}-$ FIns. 
$7 * \mathrm{P}<0.05 . * * \mathrm{P}<0.01$. 
Table 6(on next page)

Correlation analysis and multiple linear regression analysis of the serum I-FABP level for all participants. 
1 Table 6. Correlation analysis and multiple linear regression analysis of the serum I-FABP level for 2 all participants.

\begin{tabular}{|c|c|c|c|c|}
\hline & \multicolumn{2}{|c|}{ Correlation Analysis } & \multicolumn{2}{|c|}{ Multiple Linear Regression } \\
\hline & $\mathrm{R}$ & P-value & $\beta$ & P-value \\
\hline Age & 0.398 & $<0.001$ & 0.248 & $<0.001$ \\
\hline Duration & 0.413 & $<0.001$ & 0.311 & $<0.001$ \\
\hline HbA1c & 0.196 & 0.001 & 0.012 & 0.843 \\
\hline FPG & -0.019 & 0.755 & - & - \\
\hline FC-pep & -0.220 & $<0.001$ & 0.030 & 0.609 \\
\hline FIns & -0.072 & 0.282 & - & - \\
\hline $2 \mathrm{hPG}$ & 0.250 & $<0.001$ & 0.045 & 0.461 \\
\hline 2hC-pep & -0.214 & $<0.001$ & 0.018 & 0.763 \\
\hline $2 \mathrm{hIns}$ & -0.075 & 0.265 & - & - \\
\hline$\Delta \mathrm{PG}$ & 0.311 & $<0.001$ & 0.122 & 0.064 \\
\hline$\Delta \mathrm{C}$-pep & -0.171 & 0.004 & 0.012 & 0.843 \\
\hline$\Delta \operatorname{Ins}$ & -0.058 & 0.392 & - & - \\
\hline
\end{tabular}

3 The corresponding model was $\mathrm{Y}=6.749+0.026 * \mathrm{X}_{1}+0.009 * \mathrm{X}_{2}, \mathrm{Y}: \ln (\mathrm{I}-\mathrm{FABP}), \mathrm{X}_{1}$ : Duration,

$4 \mathrm{X}_{2}$ : Age, and $\mathrm{R}^{2}=0.232$ in the multiple linear regression. I-FABP $=$ intestinal fatty acid-binding

5 protein, $\mathrm{HbA1c}=$ hemoglobin A1c, FPG = fasting plasma glucose, $\mathrm{FC}$-pep = fasting C-peptide,

$6 \quad$ FIns $=$ fasting insulin, $2 \mathrm{hPG}=2$-hour plasma glucose, $2 \mathrm{hC}$-pep $=2$-hour C-peptide, $2 \mathrm{hIns}=2$ - 
7 hour insulin, $\Delta \mathrm{PG}=2 \mathrm{hPG}-\mathrm{FPG}, \Delta \mathrm{C}$-pep $=2 \mathrm{hC}$-pep - FC-pep, $\Delta$ Ins $=2 \mathrm{hIns}-$ FIns. $\beta=$ 8 standardized $\beta$-coefficient. 